\title{
A "Labyrinth" toy for 6-7 Years Old Children as a Way of Intellectual Development of a Child
}

\author{
E.E. Kozachenko ${ }^{1}$, E.M. Davydova ${ }^{1}$ \\ eek12101995@gmail.com|davydova@tpu.ru \\ ${ }^{1}$ Tomsk Polytechnic University, Tomsk, Russia
}

\begin{abstract}
The article considers the problem of a child's quick loss of interest in a toy and its solution by creating an intellectual toy based on the experience of scientific research in the field of labyrinthology. Passing puzzles in the form of a maze have a beneficial effect on the child's intellect, develops spatial thinking, memory and teaches the child how to find solutions to problems at a subconscious level, also disciplines and develops concentration. For a hull development the art design method was applied. A prototype of the designed toy is a cryptex - a portable storage used to hide secret messages invented by Leonardo Da Vinci and implemented by Justin Kirk Nevinson. The labyrinth system is based on the fractal tree method. The fractal labyrinth has a hierarchical (grid) structure, which creates more chances for a quick passage of the labyrinth without obstacles, but since the goal of the toy is to develop the intelligence of the child, it was decided to complicate the structure of the labyrinth by adding dead-end branches and additional tracks. Entering a dead-end branch allows the child to find and analyze the optimal moves in the game.
\end{abstract}

Keywords: labyrinths, children toy, design, intellectual development.

\section{Introduction}

Currently, the production of children's toys is diverse, thanks to the advent of new materials and technologies. Store shelves abound with all sorts of novelties. Every day new toys are created, which is directly related to the child's ever-increasing desire to learn the world through the game, which in turn becomes a problem for parents, because buying a new toy is costly. Some of these products do not always affect the intellectual development of children, which characterizes the toy as a useless thing.

Based on the rapid loss of interest in the old toy and the lack of intellectual development of the child while playing with it, it was decided to create an object for the game that would not only interest the child for a long time, but also allow it to develop.

Based on the foregoing, objectives were set:

Objectives of the study:

- Review and analyze existing educational toys with labyrinths;

- Examine information on the effect of labyrinths on child development;

- Collect and analyze information in the field of labyrinthology.

Design objectives:

- Develop the shape and mechanism of the toy;

- Choose a color scheme;

- Develop a system of labyrinth moves based on research in the field of labyrinthology.

\section{The passage of labyrinths as a way of developing intelligence in children}

As an idea for developing a children's toy, a maze was chosen.

The maze is a complex structure of interconnected paths. This term is also used to refer to graphic puzzles that are depicted as a network of paths and moves on a plane.

In the XX century, the maze motif is used in advertising, computer games and films. Thus, the labyrinth made a complete revolution - from the Bronze Age to Age of Computer.

The reasons for creating labyrinths are varied, but the main, at least in antiquity, was usage in various spiritual and philosophical practices. The fact is that the passage of the labyrinth was considered as the acquisition of a certain religious experience or made it possible to activate latent mental abilities by solving this puzzle.

The passage of the labyrinths has a beneficial effect on the development of logic, spatial thinking and memory of the child [1].

During the game, the child learns to find ways to solve the tasks, remembers the options for passing and gradually gets accustomed to independence.

The passage of the labyrinths contributes to the development of the ability to focus purposefully, find the right path, look back, and sometimes go back to find the shortest path. Working with labyrinths improves the quality of arbitrary attention in children: gradually increases its volume, improves its distribution, switching and stability [3].

According to the results of the study by psychologist M. Yuknat, the successful completion of the labyrinth tasks contributes to the formation of a higher level of requirements, but if the labyrinth is complex or does not have the right solution, it can negatively affect the level of claims of the child.

Emotions have a big impact on human behavior in a failure situation. In the work of I.A. Vasilyev, it is noted that in case of failure, the strategy for solving the problem changes: state-oriented people, because of experienced negative emotions, tend to solve problems in the future sequentially - analytically (which, according to experimental data from the author, is less effective) [10]. Action-oriented people do not experience negative emotions, their strategy for solving problems is holistic and intuitive, aimed at identifying the semantic context, understanding the general idea of finding a solution.

It should be noted that the labyrinths require certain skills and abilities from children, so it is senseless to give 
children under 3 years old most of them. And children will be able to evaluate some options for such games no earlier than after 9-10 years. Therefore, while choosing a maze game for their child, parents should pay attention to the age recommended by the manufacturer [2].

A toy that is not suitable for intellectual development of a child does not give an impetus to the development of logic. In the worst case, the toy can negatively affect the emotional state of the child, since too complex labyrinths can cause rejection of the game and make the child to be depressed.

\section{Designing the shape of a child's toy}

While creating the shape of a children's toy, the art shaping method was used.

The design process is the sequential execution of operations from an idea to a design project.

The main factor determining the design process is the goal of the project. The content and direction of the work of the artist-designer, the methods and means used in the process of work depend on this. The project is aimed at the properties and functions of products necessary and useful to society. The artist must design a thing so that it surpasses its analogues in utility. The design process is divided into stages, each of which has its own tasks.

In engineering or technical design, the material basis of a product is developed, and in artistic design this basis is endowed with ease of use and beauty. Thus, having other tasks, the method of artistic design is different from engineering design. The participation of the artist-designer in the development process is especially important at the beginning of the work - at the stages of the pre-project analysis and development of proposals for art design, that is, when the idea and design of a future product is born. At subsequent stages, the plan is developed and actually implemented in the project.

At the first stage of development, a systematic search for a solution occurs, the design process begins long before the drawings. At the first stage the requirements associated with the functioning of the product are determined and the general requirements of technical aesthetics are established, which always provide an integrated approach to the designed product.

At the second stage, having received the assignment, the artist-designer proceeds to a development of preliminary options of design proposals. This process is conducted in parallel with an in-depth analysis of the initial design situation and prototypes.

In the third stage, closely related to engineering design, the overall structure and design of the object is determined. The artist-designer performs a preliminary search for the future product, based on the decisions made on its design, manufacturing technology and ergonomic research data.

At the fourth stage, a technical project is being developed. Previously adopted outline design is being finalized. Knots and structural elements are developed, feasibility and implementability of the proposed solutions are checked, the layout scheme is analyzed from an engineering point of view.

The final stage - working design. At this stage, working drawings of the product are developed. The formation issues have already been resolved at the previous stages, and the scope of work of the artist-designer has been drastically reduced.

The prototype for a creating toy is Cryptex.

The cryptex presented in the «Da Vinci Code» novel is described as a stone cylinder containing "five marble discs that were folded and attached to each other in a thin brass axis. End caps do not allow you to look inside the hollow cylinder. The entire Latin alphabet is carved on each of the discs. The disc can rotate independently of other discs to create different combinations of letters and display the password [6].

The cryptex works very much like a bicycle code lock, and if someone arranges the disks correctly when entering the password, the toggle switches inside the cryptex are aligned and the whole cylinder moves apart. Inside the cryptex's internal compartment, secret information recorded on a roll of thin papyrus can be hidden. As a security measure, it can be wrapped around a fragile bottle of vinegar - if someone does not know the password, but tries to open the cryptex, the bottle breaks and the vinegar dissolves the papyrus before it can be read.

There is currently a physical implementation of the puzzle created by Justin Kirk Nevinson in 2004. Cryptex is a registered trademark of its distributor.

Figure 1 shows the «Cryptex» casket-cache.

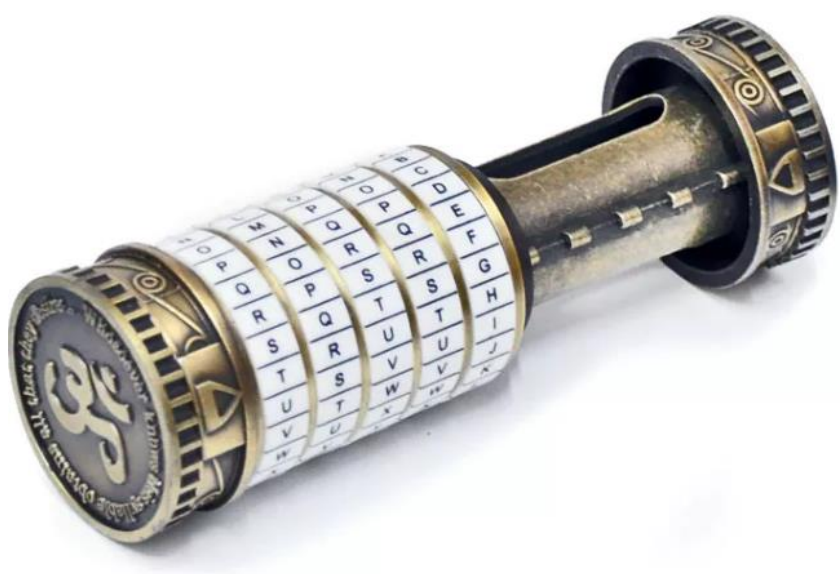

Figure 1. Cryptex (expanded)

In the designed children's toy, the principle (mechanism) by which a secret code is introduced was borrowed, but not the idea of Cryptex to protect secret information. Based on this, the mechanism of a children's toy was simplified from 5 to 3 cylinders located on one axis. Each cylinder is a network of paths, that is, a labyrinth along which a small ball pass. In Cryptex, the letters are joined together in a row, forming a code, as well as the maze cylinders create a passage for the ball.

Figure 2 shows a sketch of a children's «Labyrinth» toy.

\section{The internal structure of the toy}

The idea of the maze is to connect the cylinders so that the ball can easily pass along these paths. Based on this, it was decided to develop a system that will fix the cylinder in a certain place, creating a step with each turn. 
A system of an elastic button and cavity is used as a mechanism; for each rotation, an elastic button (button with a spring) enters the cavity, thereby fixing the cylinder and setting the step.

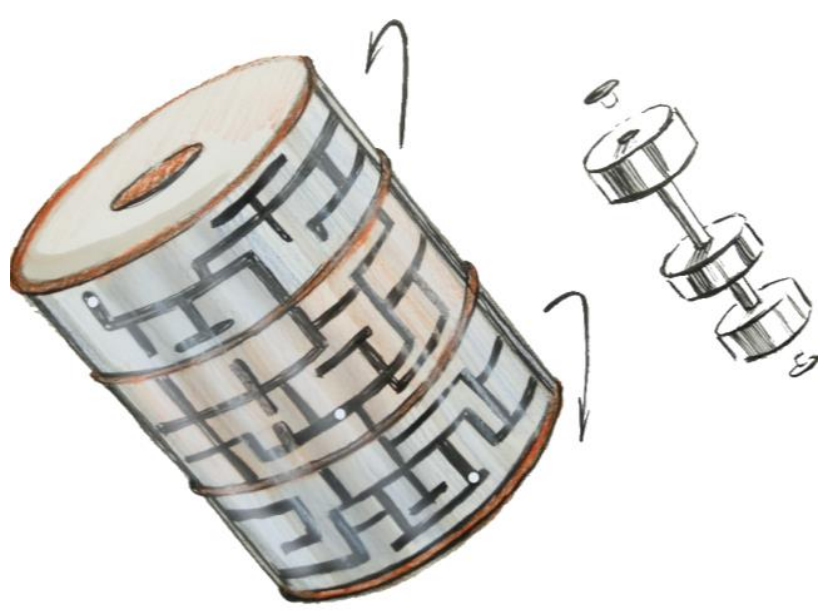

Figure 2. Sketch of the "Labyrinth" toy

To ensure that the cylinders do not touch each other too tightly and do not wear out, a rubber gasket was laid between the cylinders. However, the distance between the cylinders prevents the ball from falling out.

Three cylinders are fastened together by an axis (rod), in the base the rod has a flat plate. This plate holds the bottom cylinder. When all three cylinders are on the axis, the rod is twisted using a special attachment (clamp), which fixes the cylinders between each other.

Figure 3 shows the structure of the «Labyrinth» toy.

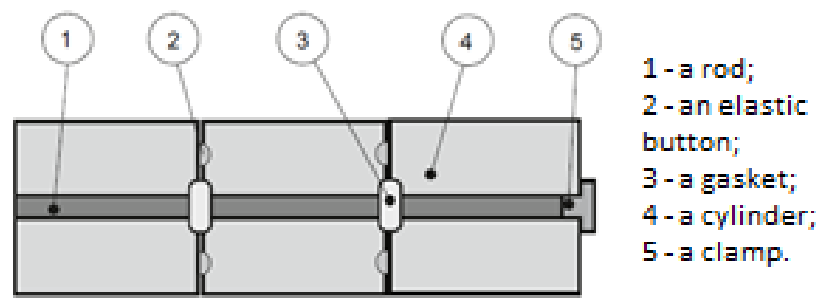

Figure 3 . The structure of the toy

\section{Color scheme}

The Labyrinth toy is positioned as an intellectual toy, so it was decided to use colors that allow you to concentrate on the game, that is, on the maze itself. The choice of color scheme was based on the legend of the Minotaur, as well as the psychological and physical effects of colors on humans.

The myth tells that in this labyrinth, King Minos hid the secret of his unfaithful wife Pasiphae, who had a passion for the sacred white bull, after which she gave birth to a monster - the Minotaur, half - a man, half - a bull. Daedalus built the Labyrinth with such intricate paths that no one could find a way out of it. Every seven years, the Athenians had to send seven young men and seven girls to the maze as a sacrifice to the monster. This has already happened twice, but the third time Theseus, the son of Aegeus, volunteered to go to the Labyrinth to fight the monster. He defeated the Minotaur and found his way back thanks to the thread attached to the entrance given to him by Ariadne, daughter of Minos.

The minotaur was a guard of the Knossos labyrinth, which was located on the island of Crete, which sandy shores are surrounded by seas [5].

Based on the foregoing, a combination of two colors was chosen as the color scheme, blue (sea) and beige (sand).

Figure 4 shows the color scheme of the «Labyrinth» toy.

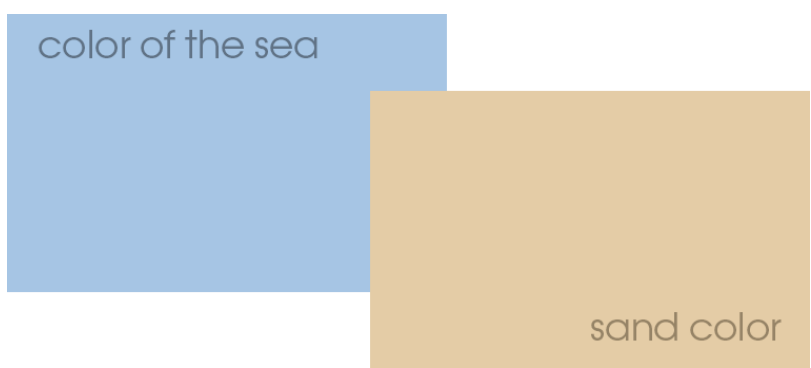

Figure 4. Color scheme

The choice of blue was due to the influence on the psychological state of the child. Blue shades help to focus on any action, especially if it is associated with remembering or studying an object. Blue color has the ability to calm and set up for work.

According to research by Max Luscher, it was found that color can affect a person both psychologically and physically.

The effect of color on a person at the physical level was determined due to a number of experiments. As a result of experiments, it was found that some colors may slightly change a person's vital indicators, such as pulse, pressure, temperature and respiratory rate.

All these changes occurred due to the influence of color on the psychological state, which in turn changed the physical indicators.

Thus, blue tones soothe, concentrate the child's attention on the toy, even out the pulse and breathing, and in some cases can lower the temperature.

Beige is a complex color, combining yellow and ocher shades. Beige, chocolate and coffee shades discipline, allow to quickly get out of an excited state [7].

\section{Design of a system of labyrinth paths}

The next step is the design of the maze itself. To implement this stage, it was necessary to do research in the field of labyrinthology.

Labyrinthology - the science of labyrinths, which includes sections such as history, types and structures of labyrinths, methods of passage, modern applications and methods of creation [4].

The first maze-like cave drawings appear on Earth in the Stone Age.

Figure 4 presents one of the oldest labyrinths carved on the wall of a tomb in Luzzanas on the island of Sardinia, built at least four thousand years ago. 


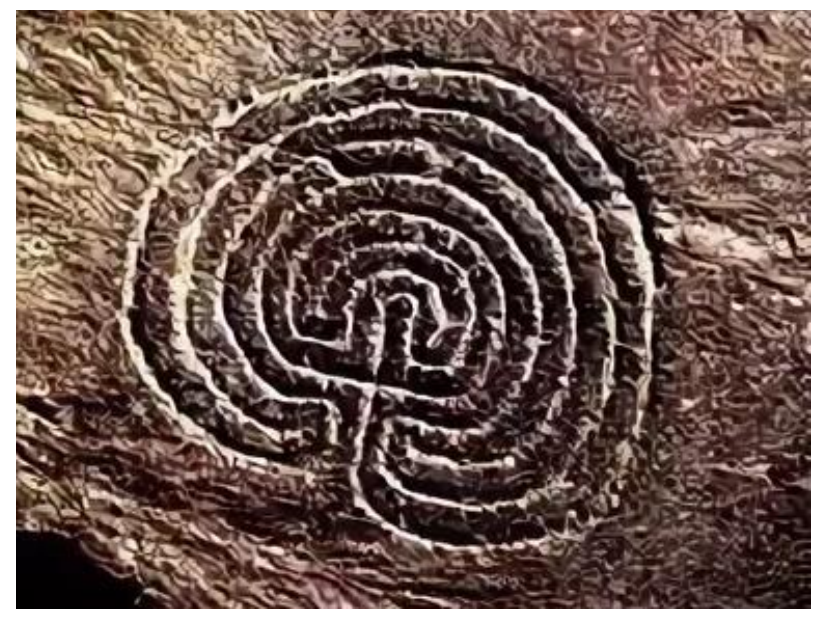

Figure 5. Image of the maze (4000 thousand years ago)

In a cultural context, prehistoric labyrinths could serve as traps for evil spirits or paths for ritual dances. Many Roman and Christian labyrinths appear at the entrances to buildings, which suggests that they served a similar apotropaic purpose (protection, reflection of evil). In a cultural study of signs and symbols, Carl Schuster and Edmund Carpenter presented various forms of the labyrinth and proposed their various possible meanings, including not only the sacred path to the house or to some goal, but also described the labyrinth itself as a master, protector and ancestor. Schuster also notes the general theme of the labyrinth, which is a refuge for the trickster: in India, the demon Ravana dominates the mazes, the trickster Jonah lives in a maze according to Sumatran bataks, and Europeans say that the maze is the house of a fraudster [8].

It can be considered that the maze is a symbol of pilgrimage, people can follow the path, rising to salvation or enlightenment. Ben Radford investigated some of the statements about the spiritual and healing effects of New Mexico's labyrinths, reporting his findings in his book «Mysterious New Mexico».

Many labyrinths were built in churches, hospitals and parks. They are often used for contemplation. Being inside the maze, among the turns, a person loses direction and connection with the outside world, thus, calms the mind and body.

In addition, the maze can be a metaphor for situations from which it is difficult to get out, like an image that is described by loss or wandering.

The fractal tree method was used to create the mazes.

The word "fractal" was introduced by Benoit B. Mandelbrot from the Latin word "fractus", which means "broken", that is, divided into parts. One of the definitions of a fractal is that a fractal is a geometric figure, which consists of parts and can be divided into multiple parts, each of which represents a reduced copy of the whole. Large-scale structures completely repeat small-scale structures.

Figure 6 shows a fractal tree scheme.

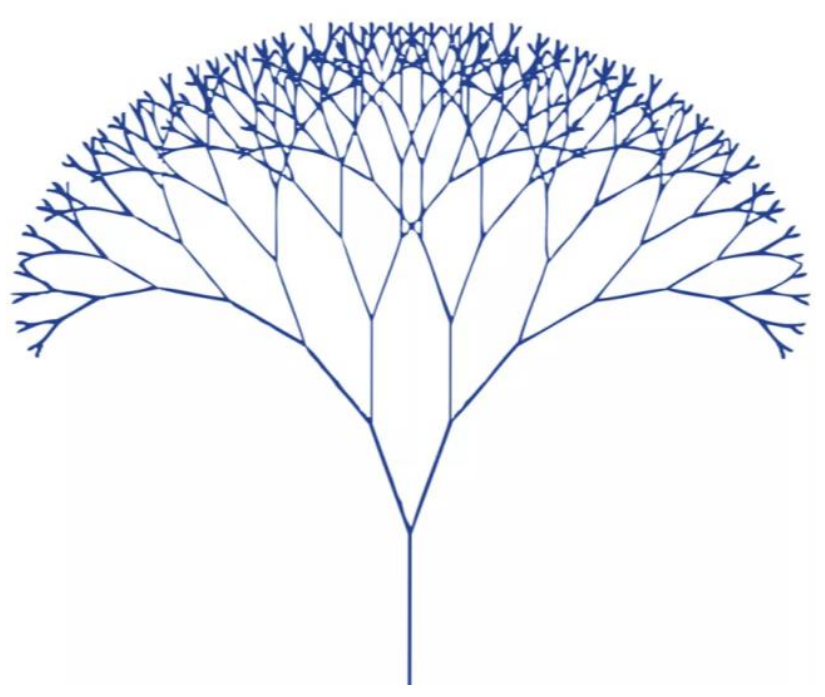

Figure 6. Fractal tree

One of the main features of fractals is self-similarity. The dimension of an object shows by what law its internal region grows. In the same way, the "volume" of a fractal increases with its size, but its dimension is not an integer value, but a fractional value. Therefore, the edge of the fractal figure is not a line, with a large increase it becomes clear that it is out of focus and consists only of spirals and curls, and the figure is repeated even on a small scale [9].

Fractals are widely used in various fields of science and technology. In computer graphics, fractals are used to create images of natural objects, such as the surfaces of the seas, trees, shrubs, mountain landscapes, etc. By using fractals, it can be able to create fairly realistic images, for example, fractals are often used to create clouds and coastlines, snow, bushes, trees, etc.

Fractals are used to create ordinary textures and background images, fantastic landscapes for computer games and book illustrations.

The maze was based on the principle of one move, followed by dividing this path into two, thereby making it possible to go through the maze in different ways for a different amount of time.

The fractal labyrinth has a hierarchical (grid) structure, which creates more chances for a quick passage without obstacles, but since the goal of the toy is to develop the intelligence of the child, it was decided to complicate the structure of the labyrinth by adding dead-end branches and additional paths. Entering a dead-end branch allows the child to find and analyze the optimal moves in the game.

Figure 7 shows the resulting labyrinths and options for their passage. The fastest passage is highlighted in red, the middle in yellow and the longest in green.

Additional passage options are created by combining the labyrinth grid and the given step of the rotations of the cylinders. 


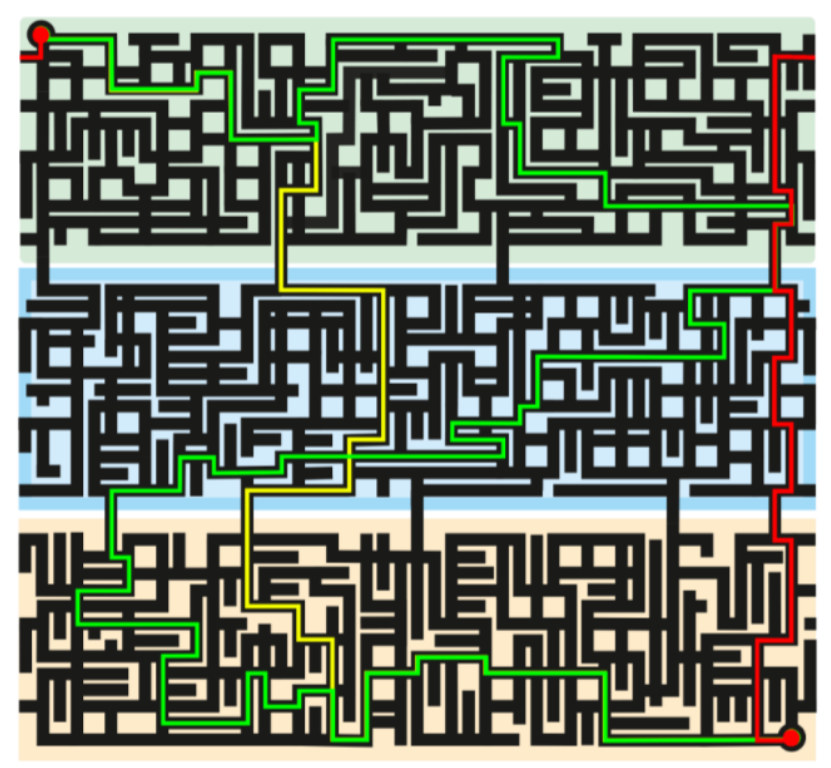

Figure 7. Labyrinth passage options

Figure 8 presents a 3D model of a children's «Labyrinth» toy.
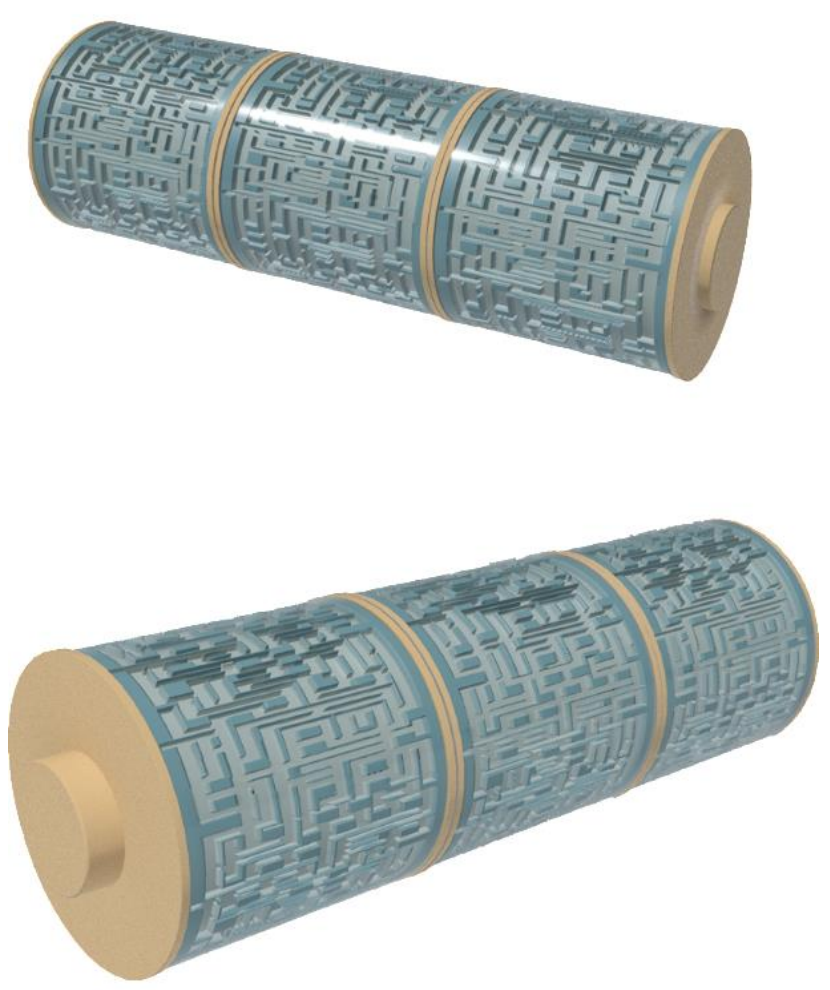

Figure 8. 3D model of the "Labyrinth" toy

\section{Conclusion}

When designing a children's "Labyrinth" toy, studies in the field of child psychology (ages 6-7 years old) were made, or rather, the types of children's toys that have a beneficial effect on the development of intelligence were studied. During the study, it was chosen to create a children's toy in the form of a maze, based on the it's beneficial effects on the child's intellect.
The information about the mazes was collected and basing on that information a unique maze was created. Passage options have been worked out.

The shape, style and structure of a children's toy was developed, taking into account the ergonomic parameters of children's hands. The dimensions of the toy are $185 \mathrm{~mm}$ in length and $60 \mathrm{~mm}$ in diameter.

At this stage, one set of cylinders with labyrinths was developed, but since the idea of the toy is long operation, it implies the development of several more sets of modules with different levels of difficulty in the passage of labyrinths (from 9-10, from 11-12 years old). So, the "Labyrinth" toy grows with the child.

\section{Reference list}

[1] Gottman, D., Dekler, D. "The emotional intelligence of a child. Practical Guide for Parents, Moscow, 2015. - 56 p.

[2] Cynthia Breazeal, Hae Won Park, Randi Williams, Stefania Druga «How smart are the smart toys? Children and parents' agent interaction and intelligence attribution» Cambridge, MA, USA, 2018.

[3] John H Flavell. «Cognitive development: Children's knowledge about the mind. Annual review of psychology», $1999,-45 \mathrm{p}$.

[4] Konforovich, A. G. "Mathematics of the labyrinths", Kiev, Radyanska school, 1987. - 144 p.

[5] Kuhn, A.N. "Myths and legends of ancient Greece", State educational and pedagogical publishing house of the Ministry of Education of the RSFSR, $1954-445$ p.

[6] Leonardo da Vinci. "Great inventions, sketches, studies", Moscow, AST Publishing House, 2019. - 320 p.

[7] Luscher M., "Luscher Color Test", Publisher: AST, Sova, $2005-33 \mathrm{p}$.

[8] Matthews, William Henry, "Mazes and labyrinths - a general account of their history and developments", University of Wisconsin, 1922г. $-357 \mathrm{p}$.

[9] Schreider M. "Fractals, chaos, power laws. Miniatures from an infinite paradise", Publisher: Regular and chaotic dynamics, 2005. - $528 \mathrm{p}$.

[10] Zeigarnik B.V. "Pathopsychology". Ed. 2nd, revised and supplemented. - M.: Publishing house of Moscow University, 1986. -287 p. 